\title{
Die meistzitierten Arbeiten des 44. Jahrgangs der Psychiatrischen Praxis - Herzlichen Glückwunsch!
}

\section{The Most Cited Articles in Volume 44 of Psychiatrische Praxis - Congratulations!}

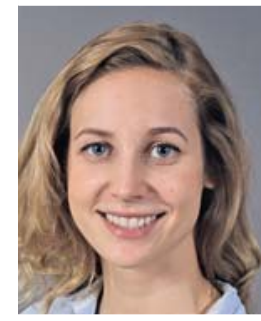

Mara-Lena Cibis

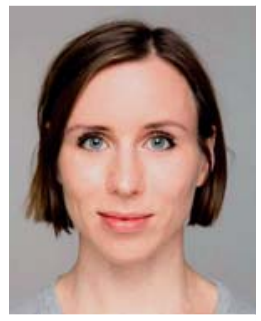

Carolin Wackerhagen
Autoren

Antje Kramer, Steffi G. Riedel-Heller

Institut

Institut für Sozialmedizin, Arbeitsmedizin und Public Health (ISAP), Medizinische Fakultät, Universität Leipzig

Bibliografie

DOI https://doi.org/10.1055/a-1033-5624

Psychiat Prax 2020; 47: 7-8

(c) Georg Thieme Verlag KG Stuttgart · New York

ISSN 0303-4259

Korrespondenzadresse

Prof. Dr. med. Steffi G. Riedel-Heller, MPH, Direktorin, Institut für Sozialmedizin, Arbeitsmedizin und Public Health (ISAP), Universität Leipzig, Medizinische Fakultät, PhilippRosenthal-Straße 55, 04103 Leipzig

Steffi.Riedel-Heller@medizin.uni-leipzig.de
Zu Beginn des neuen Jahres möchten wir an dieser Stelle wieder die meistzitierten Arbeiten unserer Zeitschrift küren und würdigen. Deutlich standen dabei die Fragen einer menschenwürdigen und patientenorientierten Psychiatrie im Vordergrund und erneut waren es Arbeiten zum Thema Vermeidung von Zwang in der Psychiatrie, die auf den vorderen Plätzen landeten. Wir freuen uns zudem, dass wir diesmal ausnahmslos Publikationen würdigen können, die in Erstautorschaft von jungen Nachwuchswissenschaftlerinnen und Nachwuchswissenschaftlern eingereicht wurden.

\section{Offene Türen}

Mara-Lena Cibis und Carolin Wackerhagen von der Klinik für Psychiatrie und Psychotherapie der Charité Berlin und Kolleginnen und Kollegen untersuchten in der mit Abstand am meisten zitierten Originalarbeit des 44. Jahrgangs, ob offene Stationstüren Aggressivität und Zwangsbehandlung reduzieren und Auswirkungen auf Entweichungsraten haben. Retrospektiv konnten sie relevante Daten einer Akutstation an der Berliner Charité vor und nach der Türöffnung auswerten und zeigen, dass bei offenen Stationstüren signifikant weniger aggressive Übergriffe und Zwangsmedikationen auftraten und sich das Entweichungsrisiko nicht erhöhte [1].

\section{Vermeidung von Zwang aus Patienten- perspektive}

Auf dem zweiten und dritten Platz finden sich gleich zwei Arbeiten, die die Betroffenenperspektive auf Zwangsmaßnahmen in der Psychiatrie und die Möglichkeiten ihrer Vermeidung in den Blick nehmen.

Kolja Heumann, Thomas Bock und Tania M. Lincoln berichteten in ihrer Arbeit von den Ergebnissen einer quantitativen deutschlandweiten Online-Befragung von Psychiatrieerfahrenen zu Erfahrungen mit Zwangsmaßnahmen und deeskalierenden Strategien [2]. Die Arbeit beschreibt das Spektrum von Zwang vermeidenden Strategien in der psychiatrischen Versorgungspraxis und zeigt, dass die Befragten eine Vielzahl milderer Maßnahmen als potenziell hilfreich einschätzen, um Zwangsbehandlungen zu vermeiden und sie das Scheitern deeskalierender Maßnahmen vor allem strukturell gegründet sehen.

Juliane Mielau und Jasmin Altunbay sowie Kolleginnen und Kollegen von der Klinik für Psychiatrie und Psychotherapie der Charité werteten teilstrukturierte Interviews mit 90 Patienten in Berliner Kliniken aus und gingen der Frage nach, welche Interventionen die Patientinnen und Patienten selbst präferieren und welche Möglichkeiten zur Vermeidung von Zwang sie im Stationssetting sehen [3]. Sie konnten zeigen, dass strukturelle Maßnahmen, wie ein größeres Angebot an Einzeltherapien, 
Verbesserungen im Hinblick auf Personalschlüssel, Ausbildung und Kommunikation sowie räumliche Rückzugsmöglichkeiten aus Patientensicht wirksame Präventionsmöglichkeiten darstellen.

Das Thema Zwang in der psychiatrischen Versorgung bleibt virulent und die Möglichkeiten und Grenzen präventiver Maßnahmen werden weiter diskutiert [4]. In der Psychiatrischen Praxis erschien eine ganze Reihe weiterer Arbeiten dazu [5-7].

Wir gratulieren den Autorinnen und Autoren ganz herzlich!

Interessenkonflikt

Die Autorinnen geben an, dass kein Interessenkonflikt besteht.

\section{Literatur}

[1] Cibis M-L, Wackerhagen C, Müller S et al. Vergleichende Betrachtung von Aggressivität, Zwangsmedikation und Entweichungsraten zwischen offener und geschlossener Türpolitik auf einer Akutstation. Psychiat Prax 2017; 44: 141-147

[2] Heumann K, Bock T, Lincoln TM. Bitte macht (irgend)was! Eine bundesweite Online-Befragung Psychiatrieerfahrener zum Einsatz milderer Maßnahmen zur Vermeidung von Zwangsmaßnahmen. Psychiat Prax 2017; 44: 85-92

[3] Mielau J, Altunbay J, Heinz A et al. Psychiatrische Zwangsmaßnahmen: Prävention und Präferenzen aus Patientenperspektive. Psychiat Prax 2017; 44: 316-322

[4] Reisch T. Reduktion von Zwangsmaßnahmen: Alles ist nicht genug. Psychiat Prax 2019; 46: 365-368

[5] Wullschleger A, Vandamme A, Ried J et al. Standardisierte Nachbesprechung von Zwangsmaßnahmen auf psychiatrischen Akutstationen: Ergebnisse einer Pilotstudie. Psychiat Prax 2019; 46: 128-134

[6] Steinert T, Scharfetter J. Wie können psychiatrische Kliniken in Österreich vollständig offen geführt werden? Psychiat Prax 2018; 45: 321326

[7] Lo SB, Gaupp R, Huber C et al. Einfluss einer „Offenen-Tür-Politik“ auf die Stationsatmosphäre: Auswirkungen auf die Behandlungsqualität. Psychiat Prax 2018; 45: 133-139 